\title{
Una possibile parabola corellana: dal paradosso amoroso rivisitato alla poesia religiosa
}

\section{A possible parable of Corella: from love paradox rivisited to religious poetry}

ANIELLO FratTA

afratta@unina.it

Università di Napoli Federico II

Riassunto: L'articolo, partendo dall'analisi dell'evoluzione della filoginia corellana, propone un possibile percorso poetico di Joan Roís de Corella: da una rivisitazione del paradosso amoroso di rudelliana memoria, che avrebbe potuto ispirare la sua concezione della ver'amor, alla poesia religiosa.

Parole chiave: Joan Roís de Corella, filoginia, paradosso amoroso, ver'amor, poesia religiosa..

\begin{abstract}
Moving from an analysis of the evolution of Joan Roís de Corella's philogyny, this paper will attempt to trace the development of his poetry: from a return to the paradox of love as formulated by Jaufre Rudel, which could have inspired his conception of ver'amor, through to religious poetry.
\end{abstract}

Keywords: Joan Roís de Corella, philogyny, paradox of love, ver'amor, religious poetry. 
Una delle acquisizioni della critica corellana degli ultimi decenni che considero più feconda è quella che riguarda gli orientamenti letterari filogini del nostro autore, con il conseguente rifiuto di tematiche e registri letterari cavallereschi o comunque portatori di sentimenti e valori eroici maschili. ${ }^{1}$ Non sembra, dunque, esagerato affermare che la filoginia è il carattere peculiare dell'opera letteraria di Corella. Essa, tuttavia, come vedremo, subisce un'evoluzione passando dalle prose mitologiche all'opera poetica profana, con uno spartiacque evolutivo rappresentato dalla Tragedia de Caldesa.

Se, infatti, la filoginia delle prose mitologiche si caratterizza principalmente per l'atteggiamento caritatevole $^{2}$ e compassionevole dell'autore nei confronti delle sue eroine, che il tempo mitologico nel quale sono vissute sottrae al giudizio della morale cattolica, ${ }^{3}$ va detto che ciò che permette loro di proporre alle altre donne la propria vicenda personale di amore-passione come un esempio ammonitorio perché non commettano gli stessi errori è il fatto che esse, cui l'autore ha ceduto l'autorità morale per poterlo fare, ${ }^{4}$ hanno la possibilità, confessando pubblicamente i propri errori, di redimersi e seppellire o superare se non proprio cancellare il proprio passato. Tutto questo facilitato sia dalla loro estraneità alla morale cristiana e quindi al concetto di peccato, sia dal loro far ricorso a un'etica laica con la sua centralità della dignità della persona e di valori imprescindibili per la donna come la pudicizia e la castità. Superare o cancellare il passato è proprio quello che non è permesso a Caldesa, caduta nel peccato, perché possibile solo a Dio («Déu no farà que el passat

1 «Caracteritzar de manera correcta el significat de l'anticavalleria de Corella és útil, doncs, sigui per matisar i precisar el vertader sentit del seu classicisme, sigui per donar l'exacte valor a la seva filogínia. Ja que l'opció de conrear una literatura filògina representa l'elecció de practicar un registre i unes temàtiques literàries oposades a les que exhibeixen valors heroics masculins, tant polítics com militars i, per tant, cavallerescos. Essent així, la crítica a la cavalleria, i als herois de la mitologia, no és, ni de bon tros, un aspecte secundari o anecdòtic del moralisme de Corella, sinó que forma part integrant, i per als contemporanis ben clara, d'una posició literària que dialoga i s'oposa a unes quantes tendències de l'època» (Cingolani 1998: 16).

2 «Sense estar-se de condemnar els errors de les seves protagonistes, és amb tendra caritat que Corella n'examina la caiguda, facilitada per la natural feblesa femenina, sobretot quan no és ajudada per la virtut cristiana, i en ressegueix la tràgica lluita moral. La consciència moral de Corella mai no vacil la; tot i així, no s'erigeix mai en moralista fustigador de la immoralitat femenina, si exceptuem els casos de Sil la i Pasifae» (Cingolani 1998: 16).

3 «No és cap amor per la classicitat i pels seus ideals el que condueix Corella a renarrar les faules ovidianes; aquestes li serveixen només per desplaçar en un remot passat unes històries d'amor-passió que, un cop ambientades en la modernitat, tindrien tot un altre significat, ja que la pagana Medea es pot compadir i els anònims protagonistes de la Tragèdia de Caldesa no. La seva filogínia no és acceptació de la luxúria o, emprant altres paraules, de l'amor; més que res li serveix per exaltar un model d'heroisme que vol ser moral i no físic o polític, ètic i no històric, i que, per tant, fora del terreny estricte de la santedat, s'expressa millor amb figures femenines, coherentment amb allò que li suggerien els seus autors. Ja que aquesta filogínia és ben coherent amb la d'uns quants models seus que individuaven l'heroisme més en el drama moral que no en l'aventura, drama que és simbolitzat, com a personatges i destinataris, per les dones possessores d'una moral íntima més veritable i menys hipòcrita» (Cingolani 1998: 16)

4 «El recurs literari de la confessió autobiogràfica, sigui una lamentació o una carta dirigida a les dones, com en les històries d'Hècuba i de Medea, desplaça l'autoritat moral des de l'autor cap a la dona que presenta per a la general edificació el seu cas tràgic, del qual és el testimoni més fidedigne» (Cingolani 1998: 17).

SCRIPTA, Revista internacional de literatura i cultura medieval i moderna, núm. 14/ desembre 2019/ pp. 229 -2 42 ISSN: 2340-4841 · doi:10.7203/SCRIPTA.14.16367 
fet no sia»); ${ }^{5}$ essa può solo perciò invocare il perdono all'amante-protagonista in nome dell'amore ed espiare le proprie colpe nelle forme che l'amante stesso richiede, compresa la morte: (vd. cit. 1)

\author{
en vós està que prengau de mi venja: \\ si us par que y bast, per vostres mans espire; \\ o, si voleu, cuberta de sçeliçi \\ iré pel món peregrinant romera. \\ Déu no farà que 1 passat fet no sia; \\ mas si sperau esmena de mon viure, \\ yo la faré, seguint a Magdalena, \\ los vostres peus lavant ab semblant aygua. (vv. 35-42)
}

Ecco perché nella Tragedia di Caldesa la compassione, che fa capolino solo nella parte finale nelle parole del narrante-protagonista, è un atteggiamento improduttivo e sterile sia per lo stato peccaminoso in cui si trova la donna, sia per la figura incarnata dal narrante, legata come vedremo alle dinamiche aberranti dell'amor cortese. Questo, tra l'altro, ci porta a ritenere la Tragedia di Caldesa come il punto di sutura tra l'ideazione e la scrittura delle prose mitologiche e l'avvio dell'esperienza poetica profana, senza che questo debba implicare necessariamente (ma logicamente lo giustificherebbe) un rapporto cronologico. Essa rappresenta il momento in cui la filoginia corellana abbandona il terreno mitologico, che sottraeva il giudizio del narratore e del pubblico alla morsa della morale cristiana, ${ }^{6}$ per tentare l'affondo nella realtà contemporanea e sfidare tale morsa. Non potendola aggirare, Corella aveva bisogno, per ricreare le condizioni che rendessero possibile la compassione e la pietà del narratore e del pubblico, di un capro espiatorio che fosse in grado se non di giustificare i comportamenti immorali della donna, almeno di attenuarne significativamente le colpe. ${ }^{7}$ Analizzando in special modo l'approccio del narrante all'ínclita donzella, con tutto il corredo topico (di topica cortese $)^{8}$ dell'ammirazione sconfinata per le sue qualità fisiche e morali e conseguente,

5 Le citazioni corellane, salvo avviso contrario, provengono da Joan Roís de Corella, Obra completa. Estudi i edició a cura de Vicent Josep Escartí; glossari a cura de Raül Hernàndez, València, Institució Alfons el Magnànim, 2013.

6 «[Corella] recorre amb riquesa extrema de detalls l'itinerari d'una ànima, el seu vertader heroisme, des del moment que, amb la pèrdua de la castedat i de l'honestedat, viola la seva més íntima i humana condició d’integritat, fins que amb el reconeixement del pecat recupera la dignitat oferint-se a la utilitat comuna. Situació, però, que es dóna, repeteixo, només si ens situem fora d'un context cristià, perquè en aquest cas l'obligació de fer-se guiar per les virtuts morals cristianes no consentiria de compadir un pecador. Medea no pot ser igual que la Magdalena, però l'una en negatiu, l'altra en positiu, representen les dues cares del mateix viatge de recuperació moral» (Cingolani 1998, p. 18).

7 Diversa l'opinione di Cingolani (1998: 16) «No és cap amor per la classicitat i pels seus ideals el que condueix Corella a renarrar les faules ovidianes; aquestes li serveixen només per desplaçar en un remot passat unes històries d'amor-passió que, un cop ambientades en la modernitat, tindrien tot un altre significat, ja que la pagana Medea es pot compadir i els anònims protagonistes de la Tragèdia de Caldesa no».

8 Cfr. anche Soler (2014: 191): «Sense descartar un possible rerefons d’irònica ambigüitat (“mos passats servirs...”), la

SCRIPTA, Revista internacional de literatura i cultura medieval i moderna, núm. 14/ desembre 2019/ pp. 229 -2 42 ISSN: 2340-4841 · doi:10.7203/SCRIPTA.14.16367 
anche espressa con linguaggio e metafora quasi sacrali, ${ }^{9}$ aspirazione a rapporto esclusivo anche fisico, ${ }^{10}$ e successivamente la profonda disperante e cocente delusione derivante dalla dolorosissima scoperta di amori plurimi della donna; e analizzando ancora il comportamento della donna che rispetta il suo ruolo di dama sans merci che lascia macerare l'amante nell'attesa; ${ }^{11}$ ci sembra di poter avanzare, sia pure con una certa cautela, l'ipotesi che il capro espiatorio trovato da Corella sia l'illusiva e ipocrita impostura dei rituali retorici dell'ideologia cortese finalizzata al piacere fisico esclusivo con la donna amata e insieme il conseguente vituperio misogino. D'altra parte la presa di posizione corellana contro l'insidia all'onestà e all'integrità delle donne rappresentata dall'apparato retorico cortese si ritrova già significativamente e accortamente presente in modo inequivoco nelle pieghe della filippica, indirizzata alle donne oneste e caste contro le arti seduttorie maschili, della protagonista di Medea:

tònica general és la d'un fidel servidor de la "senyora" en clau de fin'amors, que sotmet la seua "voluntat" a la dama, que l'enalteix com si la condescendència d'aquesta posara al seu abast la glòria del paradís (Garriga: 1994: 88): "La veu del narrador (...) parla d'uns amors que, si no sempre són honestos, són almenys ennoblits pel bany de la cultura: sobretot de la cultura literària, que refusa el tractament massa obert de determinats temes. Corella es posa del tot en aquesta banda, però sense ocultarnos les possibilitats de l'altra". És a dir, que el vincle de la parella fóra també de naturalesa sexual. "Fengia la bella senyora...", en qualsevol cas, a l'estil de les dames occitanes. Raó fonamental per la qual dedueix Carles Garriga (1994: 90) que l'escena innocent i tòpica de l'enamorat reposant sobre la falda de la seua midons»».

9 Non convince, perciò, la connotazione di erotismo un po' crasso che si suole attribuire all'espressione metaforica corellana desitjat estrado de la sua falda («una ínclita donzella [...] delliberà, aprés que en son servir molt temps de mon adolorit viure despés tenia, los meus cansats pensaments, ensems ab ma persona, en lo desitjat estrado de la sua falda descansassen»): cfr., per esempio, Annicchiarico (1991-92: 73, nota 41), che pure traduce in modo convincente la metafora: «rifugio del suo grembo, traduco così non senza qualche perplessità la metafora estrado de la sua falda. La voce estrado [...] fa parte delle predilezioni lessicali corellane: [...]. Importante, mi pare, il riapparire della stesa metafora, qui di ingente pregnanza erotica, ne la Istòria de Santa Magdalena: "la gloriosa Magdalena pres en lo estrado de la sua falda a la sacralissima sobre totes les del mon dolorosa Mare" [...] come pure interessante è il ricorrere della medesima immagine in Isabel de Villena: "La falda vostra li serà strado e cadira"»».

10 Questo si può desumere soprattutto dalla presumibile convinzione del locutore circa la verginità della donna, implicita nell'utilizzo del termine donzella, su cui cfr. Soler (2014: 192): «Independentment de les lectures arriscades que hom vulla fer, quan l'autor continua el relat sobre "la tan estimada donzella" sembla voler donar a entendre al lector que la considera verge, donzella en cabells, que exhibeix sense vel ni pudor la seua innocent bellesa juvenil, la seua "vàlua" i “estima”. Quan una dama és estuprada, cau en la depravació o es casa (esdevé "dona d'honor", en el vocabulari de l'època, al qual recorre Corella; dueña, en textos castellans), deixa de ser i de dirse donzella, denotatiu de virginitat. L'expressió jurídica i popular castellana doncella en cabello virgen, que Joanot Martorell adapta i empra en Tirant lo Blanc (Martorell 2008: 124: “totes les dones de la ciutat isqueren de la ciutat descalces; les donzeles en cabells, fent professó”) resulta incompatible semànticament amb el sobreentés d'una relació sexual».

11 Cfr. Annicchiarico (1991-92: 62): «Continua a muoversi come bella senyora e avisada senyora, impeccabile nel rispetto di un copione che le impone di mostrarsi sans merci per qualche tempo prima di accettare il servigio d'amore»; cfr. anche Soler 2014, p. 192: «La presentació de Roís de Corella, llegida per un historiador, no deixa lloc a molts dubtes: «una ínclita e bella donzella, en bellea sens par, en avisament passant totes les altres, ab gràcia e singularitat tan extrema que seria foll qui en sa presència alguna altra lloàs en estima de tanta vàlua.» I el comportament honest de la interessada s'ajusta als paràmetres d'una educació cortesa, de filla de noble o de cavaller».

SCRIPTA, Revista internacional de literatura i cultura medieval i moderna, núm. 14/ desembre 2019/ pp. 229 -2 42 ISSN: 2340-4841 ·doi:10.7203/SCRIPTA.14.16367 
O, mansuetes, púdiques, onestes e castes dones! Apreneu en guarda de vostra stima pudicícia e mirau les paraules de Jàson, de quanta honestat acompanyades, que no vol de mi cosa alguna, sinó en lícit matrimoni! Mirau ab quanta fermetat los déus invocant pren en testimoni! Mirau de quanta amor és sobrat, que la mort ab mi per millor elegeix, que, apartat de mi, viure, e veureu que, molt temps no passarà, més affectadament de mi ‘s volria partir del que ésser ab mi desiga. No sé que és pijor: o que fictament tals paraules diga, o que, dient-les ab veritat, de haver-les dites se penida. Aquesta és la comuna conditió dels variables e fictes hòmens. D’on se sdevé que res cert de ses promeses sperar és, en vanes e folles sperances, cerquar repòs en la mar furiosa. Ni m'és posible comanar a l'inanimat paper la humil continença, los lacrimants sospirs, los enamorats sguarts, ab les quals cosses axí les sues paraules acompanyada, que era quasi imposible qualsevol cosa que demanàs li fos negada (Martos 2001: 229).

Per alludere a tale apparato, Corella utilizza uno dei topoi più abusati e più retoricamente efficaci della tradizione lirica trobadorica (la preferenza, da parte dell'amante, della morte vicino o con l'amata anche riluttante alla vita lontano o senza di lei $)^{12}:$ «Mirau de quanta amor és sobrat, que la mort ab mi per millor elegeix, que, apartat de mi, viure, e veureu que, molt temps no passarà, més affectadament de mi 's volria partir del que ésser ab mi desiga». Addirittura la proposta di matrimonio avanzata da Giasone (« [...] mirau les paraules de Jàson, de quanta onesta acompanyades, que no vol de mi cosa alguna, sinó en lícit matrimoni!») può farsi rientrare nelle strategie seduttorie cortesi, anche se più proprie del romanzo cortese francese. ${ }^{13}$ Qui forse non è disutile fare un breve excursus in terra italiana, dove troviamo un illustre precedente di messa sotto accusa dell'ideologia cortese e della sua applicazione o attuazione poetica come strumento sofisticato di seduzione delle donne e fucina di trappole («ché fabricate / ho rete mante e lacci a voi lacciando》) e di lacci e lacciuoli per indurle in tentazione e cedere alle lusinghe e alle voglie dei fabbricanti di tranelli. Parliamo di

12 Cfr., per esempio, Aimeric de Belenoi, Cel que promet a son coral amic (BdT 9.8), vv. 27-28: « pero, mais voil lai morir ses doptanza / que viure sai en tan greu malanansa»; Aimeric de Peguilhan, Ara parra qual seran enveyos (BdT 10.11), vv. 19-20: «que 1 viures sai, qu'es morirs, non es gens, / e 1 morirs lai, viures sades, plazens»; Guiraut de Calanso, Bel semblan (BdT 243.5), vv. 89-92: «E si m fauc ieu totz volontos / ab fin cor gai et amoros, / car trop vueill mai morir per vos / que de nuill'autra poderos»; Lanfranc Cigala, Anc mais nuls hom non trais aital tormen (BdT 282.1c), 25-28 «Pero mais voil morir ad esien / e tornar vos vezer, que esser sors / ses vos de mort, ni viure de joi blos, / c'aital vid'es de mort piech per un cen»; Pons de Capdoill, Tuit disen q'el temps de pascor (BdT 375.25), vv. 28-30: «Mais voil morir qe m vir aillor, / q'uns francs esgartz, doutz e plazen, / que 'm feiron sei bel oill rizen»; Ponson, Ben dey viure tostemps am gran dolor (BdT 381.1), vv. 33-36: «Si l sieu valors breumens non mi secor / ben vuelh sapcha que ieu mezeis m'azir, / e lais donney, e vuelh trop mais morir / que si, vivent, vivia sens s'amon»; Rigaut de Berbezilh, Atressi con Persavaus (BdT 421.3), 21-22: «mais am per vos morir / que d'autr'aver nuill ioi, tan vos desir»; Serveri de Girona, Pus Amors vol qu'eu faça sa comanda (BdT 434a.49), 24-26: «Doncs, ma dona, mays am ades morir / que si sens joy e sens amor vivia, / e sens vos, c'am mays que re e desir» (tutte le citazioni trobadoriche provengono da Concordance de l'Occitan Médiéval. COM 2: Les Troubadours. Les Textes Narratifs en vers, Direction Scientifique: Peter T. Ricketts, Direction Technique: Alan Reed, Turnhout, Brepols, 2005).

13 Cfr. Frappier (1959: 144): «Mais cet autre "amour courtois” ne gravite pas exactement dans le même orbite que la fin'amor. Il tend à se concilier avec la morale traditionnelle, à preserver les exigences de la loi sociale et de la religion. C’est ainsi quil n'apparait nullement comme incompatibile avec le mariage».

SCRIPTA, Revista internacional de literatura i cultura medieval i moderna, núm. 14/ desembre 2019/ pp. 229 -2 42 ISSN: 2340-4841 · doi:10.7203/SCRIPTA.14.16367 
Guittone d'Arezzo accomunato a Corella, almeno nella fase palinodica, ${ }^{14}$ dall'essere un uomo di fede. Di quest'ultimo, infatti, sappiamo che fu nominato maestro in teologia tra novembre 1468 e febbraio 1471, titolo che gli permetteva di predicare ma non impediva il matrimonio; mentre di Guittone, vissuto due secoli prima di Corella, sappiamo che intorno al 1265 aderì all'ordine dei Cavalieri di S. Maria gloriosa, un ordine insieme religioso e cavalleresco, con un carattere laicale e una spiccata propensione politica. Questa è presumibilmente la data da considerare come terminus post quem per la composizione della canzone XLIX (Altra fiata aggio già, donne, parlato), che nei primi 16 versi, collegandosi all'altra canzone in difesa delle donne (la XX, Abi lasso che li boni e li malvagi), scritta quando «si trovava in una situazione di "amore" [...], si chiede se tacerà [...] ora che si trova in una condizione di "disamore" (vv. 1-4). Egli si domanda, in altre parole, se in questa fase della sua vita, connotata dal rifiuto dell'amore cortese, sia preferibile non comporre una canzone analoga a quella fatta in gioventù. La risposta è ovviamente negativa. [...] la motivazione sottostante alla canzone è sempre quella palinodica: il poeta ha infatti contratto nei confronti delle donne dei debiti troppo pesanti in passato per non avvertire la necessità di ripagarli tutti nel presente (vv. 5-6). L"'onore" e il "piacere" che egli ha preso dall'esercizio della fin'amor vengono pertanto smascherati, rivelando la loro vera natura negativa e distruttiva, la loro essenza di “onta” e "gravezza” (vv. 7-8)» (Picone 1995: 87):

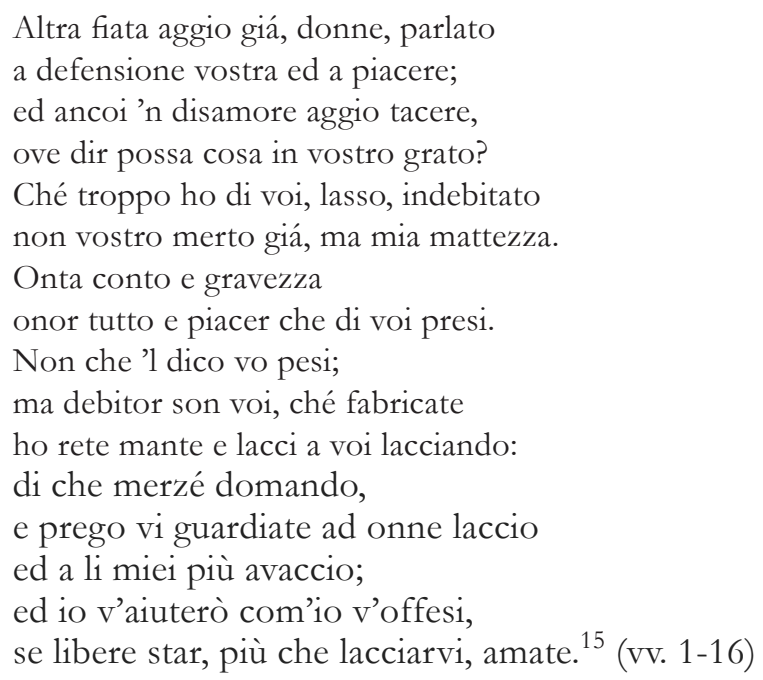

14 «Le canzoni XX, Ahi lasso, che li boni e li malvagi, e XLIX, Altra fiata aggio già, donne, parlato, adiacenti nel codice Laurenziano Rediano 9, si propongono entrambe lo scopo della defensione delle donne contro l'inganno e la falsità del modello "cortese"; inoltre, la seconda costituisce una vera e propria palinodia di alcune fra le posizioni assunte dal Guittone "amoroso" (o prima maniera)» (Poli 2000: 95).

15 Si cita da Egidi (1940: 131), con ritocchi ai vv. 3-4.

SCRIPTA, Revista internacional de literatura i cultura medieval i moderna, núm. 14/ desembre 2019/ pp. 229 -2 42 ISSN: 2340-4841 · doi:10.7203/SCRIPTA.14.16367 
Conseguente a tale denuncia bruciante è l'appello alle donne a mantenersi oneste e caste (che rappresenta una variante paternalistico-didascalica dell'astinenza crudele predicata da Corella). E la cosa sorprendente è che in Guittone troviamo lo stesso aggettivo ('mansueto'), oltre all'identico invito a mantenersi caste e pudiche, usato da Corella:

\author{
Conven con castitate a donna avere \\ umilitá, mansuetudo e pace: \\ figura mansueta non conface \\ orgoglio asprezza e odio alcun tenere. (vv. 131-34) (Egidi 1940: 134).
}

Ma ancora più sorprendente è la quasi sovrapponibilità dell'invito che Medea rivolge alle donne caste e pudiche e mansuete a diffidare delle dichiarazioni e delle promesse d'amore eterno, false o volubili, accompagnate anche da sospiri e lacrime, che un uomo, sulla falsariga di Giasone, potrebbe rivolgere loro, con la durissima diffida guittoniana:

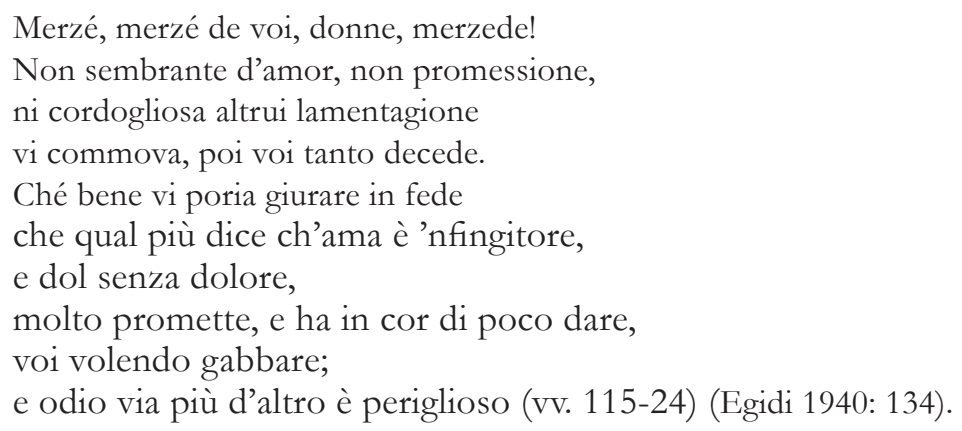

Un più lungo discorso andrebbe fatto su Boccaccio e in special modo sull'Elegia di Madonna Fiammetta; ma lo rimandiamo ad altra occasione.

Ritornando alla Tragedia di Caldesa e alla denuncia dell'impostura dei rituali retorici cortesi in essa supposta, è addirittura possibile affermare che Corella abbia inteso dare una drammatica veste narrativa a tale impostura, egregiamente rappresentata dal cant LXXI di Ausiàs March. ${ }^{16}$ Questa importante poesia doveva costituire per Corella l'esempio più recente e più luminoso dell'illusione cortese di un rapporto anche fisico esclusivo con l'amata, illusione che, messa di fronte alla realtà ben diversa delle cose, sfocia, per la delusione patita, in un misoginismo quasi antropologico. Ma l'atteggiamento diverso dell'autore e del protagonista-narrante rispetto al locutore di Què m'ba calgut contemplar en amor si evidenzia già dal titolo dell'opera: la vicenda narrata è una "tragedia" non solo

16 Cfr. Cingolani (1998: 21): «Sintetitzant al màxim, podríem dir que l'acte d'escriptura de Corella és determinat per la voluntat de donar un cos narratiu a experiències de tipus líric, com assenyalava Lola Badia. Més en concret, es pot percebre aquest esquelet líric com d’inequívoca ascendència ausiasmarquiana, mentre que la polpa la dóna la mitologia clàssica». 
perché racconta la fine drammatica di un'illusione e di un'impostura, ma perché questa impostura illusiva ha comportato l'annientamento morale di una creatura (Caldesa) che avrebbe meritato un destino ben diverso, avendo lei in potenza tutte le qualità, fisiche e spirituali (queste ultime risaltate dalle humils paraules che ella rivolge all'amante deluso) per diventare un esempio fulgido e un alto modello di donna supremamente bella e incorruttibile. Tale atteggiamento, tuttavia, almeno riguardo al protagonista narrante, si incrina nelle righe finali dell'opera: qui, infatti, egli, pur provando una vera compassione per la donna, compassione prestatagli dall'autore, ma incarnando al contempo l'ipocrita intransigenza dei cultori e praticanti dell'amor cortese, può solo dolersi sanguinosamente dell'irreparabile 'tragedia' che ha coinvolto l'ínclita donzella e affiancare al rammarico di non poter cancellare il passato il desiderio apparentemente irrealizzabile di una scissione della parte buona della donna (la sua gentil persona ab tan subtil enteniment), da lui ammirata, da quella 'cattiva' (la sua falla e moble voluntat, de falsa estima guiada), che sarebbe stato più giusto albergasse in un cos lleig e diforme:

Si follia és començar lo que és impossible fi atenyga, folla cosa seria assajar escriure los contrasts que ma dolorosa pensa combatien, aprés d'haver dit resposta de tan humils paraules. Volguera, ab preu de ma vida, la sua tan gran erra se pogués rembre. Oh, quant estimara beure de l'aigua del riu Letes, perquè, lo passat absent de la memòria, sol en lo present atengués mon enteniment terme! E fóra més alegre, aquesta bella senyora en parts de singular partida, la sua gentil persona ab tan subtil enteniment fos la part mia, e la sua falla e moble voluntat, de falsa estima guiada, cercàs un cos lleig e diforme, en part d'aquell qui indignament l'havia tractada.

La scissione si realizzerà, invece, nella sua opera poetica profana e si tradurrà in una scissione tra la figura della donna 'perfetta' che possiede i requisiti necessari per costituire la rappresentante ideale della vera amor e la femmina diventata puro oggetto di piacere, provocando la sparizione totale dell'atteggiamento compassionevole dell'autore. La scissione si rifletterà anche sulle caratteristiche che assumerà la produzione poetica di Corella, dando vita a una divisione netta tra poesie di ammirazione (propriamente adorazione) e 'lode' della donna crudele e incorruttibile e perfetta da quelle di maldicenza (veri e propri maldits) rivolte a Caldesa, quale rappresentante delle donne moralmente corrotte e degradate. Nell'ideologia amorosa corellana, ricavabile dalle sue poesie profane, solo la donna può conservare la purezza e la bellezza sublime dell'amore buono e vero (la bon'amor/vera amor) e al tempo stesso la sua immacolata assolutezza, fatta di onestà e fedeltà, erigendo una impenetrabile barriera a ogni contaminazione fisica mediante lo strumento della crudeltà e della strenua e invincibile resistenza a ogni lusinga o tentazione da parte dell'amante. Infatti quando ella cede alle lusinghe amorose (anche quelle raffinate della fin'amor), il suo destino è segnato ed è quello che simbolicamente si può cogliere, come si è visto, nella vicenda di Caldesa raccontata nella Tragedia di Caldesa, anche se l'umana simpatia di Joan Roís per la donna e il suo atteggiamento indulgente nei confronti delle sue colpe erompe nel finale con toccante delicatezza. Questo ci induce a ritenere che nell'elenco dei modelli letterari di Corella stilato da Cingolani, «que individuaven l'heroisme més en el drama moral que no en l'aventura, drama que és simbolitzat, com a personatges i destinataris, per les dones possessores d'una moral íntima més veritable i menys 
hipòcrita», ${ }^{17}$ manchi un referente filogino importante, vale a dire la lirica cortese nella sua versione più pura e dura, quella originaria, rudelliana soprattutto, ancora condizionata dall'idea di peccato e di tralignamento morale implicito nell'amore carnale.

Sicché, in definitiva, l'ideologia amorosa corellana potrebbe, a nostro parere, considerarsi come una rivisitazione aggiornata e, per certi aspetti, tragica del "paradosso amoroso" spitzeriano ${ }^{18}$ nella versione rudelliana dell'amor de lonh. Con marcate differenze. Mentre, infatti, in Jaufre Rudel è l'attesa indefinibile e infinita dell'amor de lonh che appaga e dà gioia e rende sostenibile il dolore dilaniante della sua irrangiungibilità, gioia che nasce dalla consapevolezza che la condizione di amante dell'amor de lonh offre la possibilità di ovviare alla regio longinqua dissimilitudinis; ${ }^{19}$ in Corella, in cui la distanza è stata sostituita dalla irremovibile e ferrea resistenza della donna, l'unica gioia e sollievo al dolore può venire solo dal pensiero dell'imminenza della morte per amore, del martirio per amore. La cosa è apertamente dichiarata nella cobla che inizia con Si en lo mal temps la serena bé canta:

Si en lo mal temps la serena bé canta, jo dec cantar, puix dolor me turmenta en tant extrem que ma pensa és contenta de presta mort : de tot l'aldre s'espanta. Mas, si voleu que davall vostra manta muira prop vós, hauran fi mes dolors: seré l'ocell que en llit ple de odors mor, ja content de sa vida ser tanta.

17 «L'Ovidi de les Metamorfosis i de les Heroides, el Boccaccio d'uns contes del Decameron i de la Fiammetta que beu a les Tragèdies de Sèneca igual que Corella- o el mateix Sèneca - que identifica la guerra amb el poder, tan nefast i tràgic -, l'induïen a resseguir aquest camí que, com a possibilitats masculines d’un drama íntim i moral, només proporcionava les típiques sofrences amoroses, tan explotades per la lírica, a l'estil d'Ausiàs March, i que sempre amagaven, per Corella, un fons d’immoralitat i luxúria» (Cingolani 1998: 21).

18 «Cet amour, entouré des brumes du rêve, [est] la manifestation la plus émouvante de ce que j’appelais le 'paradoxe amoureux' qui est ä la base de toute la poésie trobadoresque: amour qui ne veut pas posséder, mais jouir de cet état de non-possession, amour-Minne contenant aussi bien le désir sensuel de 'toucher' à la femme vraiment 'femme' que le chaste éloignement, amour chrétien transposé sur le plan séculier, qui veut have and not have» (Spitzer 1944: 1-2).

19 Cfr. (Gubbini 2014: 892): «Analogamente al peso della corporeità che allontana dalla somiglianza con Dio e trasporta l'anima nella regio longinqua dissimilitudinis, la cupidigia sregolata (verso altri amori o verso la stessa Midons) vanifica il desiderio e l'aspirazione al raggiungimento dell'amor de lonh. L'amor de lonh richiede infatti la saggezza dell'attesa [...], la resistenza strenua al dolore che sana con gioia [...]. Nel tema della regio dissimilitudinis, una volta superato questo ostacolo, l'uomo è restaurato nella primitiva somiglianza con Dio, e torna (revertere) al Sommo Bene [...]. In termini analoghi anche Jaufre esulta di gioia in Belhs m'es l'estius; è restituito (restauraty) al suo valore e non andrà mai più altrove [...]. Attraverso la disamina di questi diversi tasselli sembra quindi di aver identificato nell'amor de lonh di Jaufre Rudel una "traccia" del tema -agostiniano, cistercense e, più in generale, monastico- della regio longinqua dissimilitudinis. Un tema, un'immagine e un lessico di derivazione monastica che, patrimonio comune dell'uomo medievale, acquistano però nella poesia rudelliana un significato "nuovo": quello della celebrazione mistica di un amore squisitamente profano». 
Questa cobla è di straordinaria importanza perché, nell'utilizzazione di uno dei topoi più diffusi della poesia cortese ("il conforto dell'uomo selvaggio" qui sostituito da quello della sirena) ${ }^{20}$, ci svela la volontà del poeta di evidenziare il legame della sua poesia (e della concezione amorosa che vi è sottesa) alla tradizione poetica europea scaturita dall'ideologia della fin'amor. L'utilizzazione del topos, tuttavia, è radicalmente rivoluzionata rispetto alla tradizione: infatti qui la speranza autoindotta dal canto non riguarda il cambiamento delle condizioni esterne (da negative a positive per il mutato atteggiamento della donna amata), improponibili e impossibili, ma l'accettazione da parte della donna del martirio per amore del locutore amante. ${ }^{21}$ D'altra parte, che il canto di quest'ultimo non abbia nessuna possibilità di scalfire la crudeltà della donna e non serva a muoverla a compassione, è confermato dalla prima cobla di És vostre cor d'acer, ab tan fort tempre, dove pure ritorna il canto delle sirene:

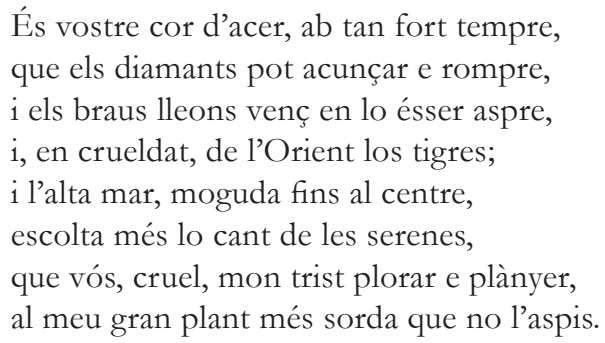

E la crudeltà della donna, alla fine, può salvare anche il locutore dalla dannazione eterna, a conferma che essa ha la stessa funzione di allontanamento dalla regio longinqua dissimilitudinis dell'amante coinvolto che aveva l'amor de lonb nell'ideologia amorosa rudelliana:

\footnotetext{
Si no poguí restaurar-vos lo viure

sol per temor de honestat ofendre, no us vull negar com aprenguí de doldre:

a Déu pregant guardàs del fondo carçre vostre esperit, que al meu era conforme. Mudarà el gest la mia forma en pedra, quan llegiran aquest mot en la tomba: «Pensant per mi, haveu aprés de plànyer». E no em doldrà la mia vida trista, que sol per vós la poguí bé despendre.
}

20 Sull'argomento cfr. Boase (2016: soprattutto 672): «In this respect, the siren's behaviour is evidently modelled on that of the wild man, who, by singing in the storm, demonstrates the virtue of hope in the chill of love's adversity».

21 Elemento questo che differenzia profondamente il locus corellano da quello che viene considerato come il suo ipotesto, vale a dire il sonetto del Marchese di Santillana En el próspero tiempo las sirenas: cfr. Deyermond (2007: 125). 
D'altra parte, però, in questo scenario sostanzialmente teologico, nel quale viene apertamente dichiarato il potere salvifico della incorruttibile e adamantina durezza della donna, Corella riesce a ritagliarsi spazi di lirismo delicato, come nei versi che precedono quelli appena citati:

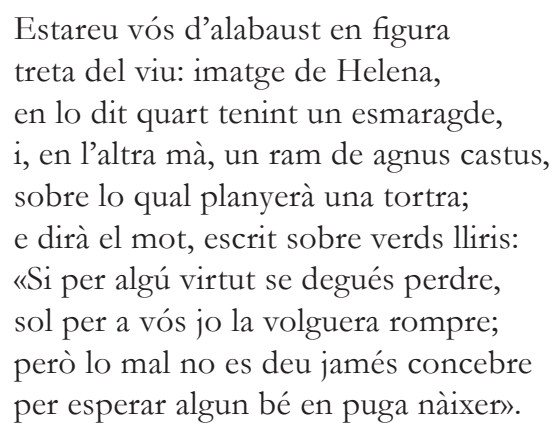

La concezione corellana del martirio d'amore come unica strada percorribile per garantire nel contempo la virtù della donna e l'allontanamento dell'amante dalla regio longinqua dissimilitudinis è lo sbocco inevitabile e inesorabile della critica di Roís de Corella alla bon'amor di Ausiàs March. Lo dimostra in modo inequivocabile lo strettissimo ed evidentissimo rapporto genetico che esiste tra la poesia LXXI (Los ignorants Amor e sos exemples) di March, durissimo manifesto del suo misoginismo con conseguente rinuncia all'amore, e Los qui amau, preneu aquesta cendra di Roís de Corella, che si può senz'altro considerare come il manifesto della concezione corellana della vera amor.

Rimandando a un mio articolo ${ }^{22}$ specificamente dedicato a illustrare nel dettaglio questo rapporto, possiamo concludere, in definitiva, che per Corella l'unica possibilità che hanno coloro che amano (Los qui amau in opposizione a Los ignorants Amor) di non perdere il consiglio della ragione e quindi di non cedere all'amore-piacere è accettare l'astinenza fino al martirio, alla morte per amore; proprio quello che, come si è visto, Corella si augura per le proprie atroci sofferenze in Si en lo mal temps.

La morte, dunque, come fine del dolore e dei patimenti amorosi, ma anche metafora della fine della sensualità nella bon'amor, dell'amore-piacere, condizione già in qualche modo delineata nel citato verso 86 di Los ignorants («ffinit aquell qui 'n tal estrem los mena») ${ }^{23}$. Il desiderio di morte, dunque, è desiderio di martirio indotto dall'annientamento del desiderio fisico, è il cupio dissolvi conseguente alla morte dei sensi, alla rinuncia all'amore-piacere. Morte dei sensi e rinuncia all'amore-piacere già attuate dalla donna destinataria dell'amore del poeta per difendere la propria onestà, difesa spinta fino alla crudeltà disumana e all'insensibilità verso il dolore dell'amante, che viene indotto anche alla morte del corpo. E il dissolvimento dei corpi dell'amante e dell'amata è il risultato inevitabile della morte dei sensi, come rivela un'altra poesia di Corella, quella che inizia con En lletres d'or tendreu en lo sepulcre:

22 «La bon'amor/vera amor in Ausiàs March e Joan Roís de Corella», in Ausiàs March: leggere, editare, tradurre nel tempo, Convegno internazionale, Napoli 26-27 novembre 2018, in corso di stampa.

23 Questo verso, infatti, a nostro parere va così interpretato: 'una volta messo fine a quel pensiero (compte) che lo conduce a tali estremi', dove il pensiero non può essere altro che il desiderio del rapporto fisico con la donna. 


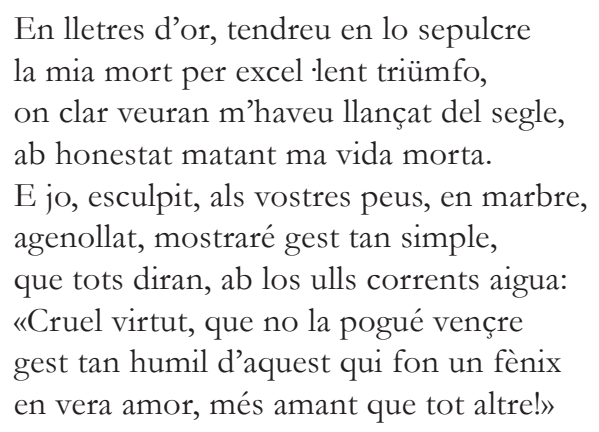

Ma la morte fisica riserva una sorpresa inattesa: la donna amata, non più obbligata alla crudeltà per difendere la sua virtù, può solo lì, nel sepolcro, recuperare la sua umanità e finalmente provare per il suo amante, un campione irripetibile e unico della bon'amor (un fenix / en vera amor), una pietas amorosa e una dolcezza improponibili in vita, fino ad arrivare addirittura a conoscere il dolore per la sua sorte, pregando Dio di risparmiare il carcere infernale allo spirito di lui, conforme al suo:

e dirà el mot, escrit sobre verds lliris: «Si per algú virtut se degués perdre, sol per a vós jo la volguera rompre; però lo mal no es deu jamés concebre per esperar algun bé en puga nàixer».

Si no poguí restaurar-vos lo viure sol per temor de honestat ofendre, no us vull negar com aprenguí de doldre: a Déu pregant guardàs del fondo carçre vostre esperit, que al meu era conforme» (vv. 16-25).

D'altra parte, però, in un'ideologia amorosa di stampo teologico come questa di Corella, l'abolizione del desiderio nel rapporto che lega l'amante all'amata crea un vuoto che il poeta cerca di colmare con l'adorazione. Anzi nel componimento Del jorn que us viu d'altra gens no em pot plaure, per confermare l'avvenuta sostituzione, viene utilizzato proprio il sintagma più famoso della tradizione trobadorica relativo al desiderio amoroso (ferm voler) per indicare il forte zelo del locutore nel culto adorante della donna (senyora):

\footnotetext{
Del jorn que us viu d'altra gens no em pot plaure sinó de vós, a qui prenc per senyora: $\mathrm{ab}$ ferm voler ma pensa en vós adora e de mon cap absència no es pot raure. Oh, folls jueus, bé mereixquéreu caure adorant déus de fust, sens tal bellea! Mas qui bé us veu, quítia de lletgea, d'adorar-vos és foll si es pot retraure.
} 
C'è in questi versi come la coscienza che l'adorazione della donna possa in qualche modo sconfinare nei territori dell'idolatria pagana, al pari della venerazione del vitello d'oro da parte dei giudei; ma quello che distingue la donna adorata dai simboli pagani non è solo la bellezza ma l'immacolatezza che, donandole per ciò stesso un'aura sacrale, la apparenta di diritto a immagini sacre. Il passo successivo nell'evoluzione della poetica corellana non poteva essere che la poesia religiosa.

\section{Bibliografia}

Annicchiarico, Annamaria (1991-92), «Perché "tragèdia"?: il gioco delle "ambiguità" nella "Tragèdia de Caldesa" di Joan Roís de Corella», Boletín de la Real Academia de Buenas Letras de Barcelona, 43, pp. 59-79.

Boase, Roger (2016), «The Siren in the Storm and the Wild Man's Solace: a Folkloric Motif in Fifteenth-Century Spanish Poetry», eHumanista, 32, pp. 668-676.

Cingolani, Stefano Maria (1998), «Joan Roís de Corella i els límits de la literatura », Caplletra, 24, pp. $13-24$

Deyermond, Alan (2007), «Las imágenes del bestiario en la poesía de Joan Roís de Corella», in Poesía de cancionero del siglo XV. Estudios seleccionados, a cura di Rafael Beltran Llavador, José Luis Canet Vallés e Marta Haro Cortés. Valencia: Universitat de València, 2007, pp 119-31.

Egidi, Francesco (1940), Le rime di Guittone d'Arezzo, a cura di F. E., Bari, Laterza (Scrittori d'Italia, 175).

Frappier, Jean (1959), «Vues sur les conceptions courtoises dans les littératures d'oc et d'oil au XIIe siècle», Cabiers de Civilisation Médiévale, 2 , pp. 135-56. 
Aniello Fratta. Una possibile parabola corellana: dal paradosso amoroso rivisitato alla poesia religiosa

Gubbini, Gaia (2014), «Amor de lonb: Jaufre Rudel, Agostino e la tradizione monastica», Dai pochi ai molti: Studi in onore di Roberto Antonelli, a cura di P. Canettieri e A. Punzi, Roma, Viella, pp. 885892.

Martos, Josep Lluís (2001), Lesproses mitològiques de Joan Roís de Corella: edició crítica, Alacant/Barcelona, Institut Interuniversitari de Filologia Valenciana / Publicacions de l'Abadia de Montserrat («Biblioteca Sanchis Guarner», 55).

Picone, Michelangelo (1995), Guittone e $i$ due tempi del "Canzoniere", in Guittone d'Arezzo nel settimo centenario della morte, Atti del convegno (Arezzo, 22-24 aprile 1994), a cura di M. Picone, Firenze, Cesati, pp. 73-88.

Poli, Andrea (2000), «Una scheda provenzale per Guittone. Le canzoni XX, "Ahi lasso, che li boni e li malvagi", e XLIX, "Altra fiata aggio già, donne, parlato"», Filologia e critica, 25, pp. 95-108.

Roís de Corella, Joan (2013) Obra completa. Estudi i edició a cura de Vicent Josep Escartí; glossari a cura de Raül Hernàndez, València, Institució Alfons el Magnànim.

Soler, Abel (2014), «Les dones, l'amor i una elegia eròtica d'Ovidi en la vida i en l'obra poètica de Roís de Corella», SCRIPTA, Revista internacional de literatura i cultura medieval i moderna, 3, pp. 178208.

Spitzer, Leo (1944), L'amour lointain de Jaufré Rudel et le sens de la poésie des troubadours, Chapell Hill, University of North Carolina Press. 